\title{
LEYES DE CRUCE EN SPINOZA. BELLEZA, LÓGICA Y NATURALEZA
}

\author{
Sara Reyes Vera \\ Universidad de La Laguna \\ sarareyes.rpf.ull@gmail.com
}

\section{RESUMEN}

Análisis del concepto de belleza como acto desde la producción de la naturaleza y sus leyes de cruce bajo la fórmula Deus sive X a partir de Deus sive Natura. Esto guía el estudio sobre las relaciones entre lo uno y sus representaciones múltiples y sus conexiones lógicas. La ignorancia y lo imposible están en este desarrollo para conocer la potencia divina y genuina de las infinitas causas para la singularidad humana. Se propone la belleza como verbo y no como adjetivo donde el par belleza/deformidad expresa forma/no-forma.

Palabras clave: belleza, Dios, Naturaleza, ignorancia, leyes, imposible, lógica, forma, deforme.

\section{LAWS OF CROSSING IN SPINOZA. BEAUTY, LOGIC AND NATURE}

\section{Abstract}

An analysis of the concept of beauty as an act of Nature's production and its laws of crossing, under the formula Deus sive X from Deus sive Natura. This guides a study on relationships between oneness and its multiple representations and logic connections. Ignorance and the impossible are in this development to understand the divide and genuine potency of the infinite causes for human singularity. Beauty is framed as a verb rather than an adjective, with the dual beauty/deformity expressing form/non-form.

KeYwords: Beauty, God, Nature, ignorance, laws of crossing, the impossible, logic, form, deformity. 
Spinoza expone su pensamiento de tal forma que adelanta una reflexión nueva sobre lo pensado. Abre perspectivas. En cada nueva expresión su filosofía se despliega por amor a la verdad a la filosofía de quien como él se mete en harina, investigando y enmendando "con un poco de meditación»" Leer el Apéndice de la primera parte de la Ética aceptando su invitación a la crítica dirige nuestra atención hacia los prejuicios. El Dios de Spinoza es un ente absolutamente infinito no depende de nada, «una substancia que consta de infinitos atributos, cada uno de los cuales expresa una esencia eterna e infinita $»^{2}$.

El filósofo Étienne Balibar estudia este asunto desde una interesante perspectiva con el propósito de resolver la paradoja implícita entre la esencial unidad del Dios spinozista y sus representaciones múltiples. La división del «objeto» de trabajo, que además relaciona con los tres géneros de conocimiento, muestra una tópica de tres dioses ${ }^{3}$ : 1) Dios «naturalista» Deus sirve Natura sive Potentia sive Causa 2) Dios «monoteísta» Deux sive Rex sive Lex 3) Dios Humanista Deus sive Amor sive Homo. En sí es el primero de los enunciados sobre Dios el que revela la potencia de la belleza como acción porque es aquí donde las leyes de la naturaleza se muestran en las causas relacionales y su traducción para el conatus. La perseverancia es aproximación al conocimiento ${ }^{4}$ por una necesidad que a veces queda insatisfecha. En ello va la pregunta construida "para exhibir el mundo como proceso" ${ }^{5}$ y aceptar la ausencia de respuesta. En ese plano se sitúa la pregunta sobre el par belleza/deformidad con

dice.

${ }^{1}$ SpInoza, Ética, edición bilingüe por Pedro Lomba, Trotta, Madrid, 2020. Cf., EI, Apén-

2 EI, def. vi.

3 Etienne Balibar, Spinoza politico. Lo transindividual, Editorial Gedisa, Barcelona, 2021, p. 268.

${ }^{4}$ En opinión de Robert Misrahi, la naturaleza como sistema lógico de determinaciones a las que recurrir para hacer progresar el conocimiento. Este es un hecho claro por el que la toma de posición crítica se hace fuerte ante la ignorancia como refugio. Sin embargo, la condición emocional que aporta el significado de guarida no queda absolutamente explicada solo desde esa perspectiva: «Todos los prejuicios que intento indicar aquí dependen de uno solo, a saber: el hecho de que los hombres supongan, comúnmente, que todas las cosas de la naturaleza actúan, al igual que ellos mismos, por razón de un fin, e incluso tienen por cierto que Dios mismo dirige todas las cosas hacia un cierto fin, pues dicen que Dios ha hecho todas las cosas con vistas al hombre, y ha creado al hombre para que le rinda culto. Consideraré, pues, este solo prejuicio, buscando, en primer lugar, la causa por la que le presta su asentimiento la mayoría, y por la que todos son tan propensos, naturalmente, a darle acogida. Después mostraré su falsedad y, finalmente, cómo han surgido de él los prejuicios acerca del bien y el mal, el mérito y el pecado, la alabanza y el vituperio, el orden y la confusión, la belleza y la fealdad, y otros de este género. Ahora bien: deducir todo ello a partir de la naturaleza del alma humana no es de este lugar. Aquí me bastará con tomar como fundamento lo que todos deben reconocer, a saber: que todos los hombres nacen ignorantes de las causas de las cosas, y que todos los hombres poseen apetito de buscar lo que les es útil, y de ello son conscientes». EI, Apéndice. $C f$., Robert Misrahi, Spinoza, Edaf, París, p. 66.

${ }_{5}$ François Zourabichvili, Spinoza. Una física del pensamiento, Editorial Cactus, Buenos Aires, 2014, p. 12. 
la misma búsqueda que plantea el par forma/no-forma ${ }^{6}$. Esto es, desde el punto de vista de la forma desplazada de su génesis pensar la belleza como acto transformador.

La Naturaleza al determinar la complejidad del modo también determina las causas de una mayor o menor perfección en función de la realidad en cada uno contenida. Lejos de reglas mecanicistas la potencia explica esa arquitectura de lo material ${ }^{7}$. Con esas coordenadas podemos hacer uso de la construcción Deus sive X a partir de la sentencia Deus sive Natura convertida por Balibar en un consecuente más amplio y "abierta a diferente posibilidades de satisfacción» ${ }^{8}$ para un entendimiento cuya específica conciencia no busca refugio en la ignorancia.

La condición bidireccional Deus sive $X$ quiere «tomar en serio lo que dicen los textos, sin descuidar las estrategias retóricas de la escritura»", en todo caso es una fórmula más compleja que tanto afecta a la definición de Dios como a la definición de Naturaleza, abierta a los infinitos atributos desde un contexto de razón predeterminado en el que no existe negación ${ }^{10}$ alguna para: la producción de lo imposible como acto de belleza, fábrica que sigue el patrón Naturaleza o X, la deformidad para esa acción de belleza que refleja la paradoja ${ }^{11}$ entre lo uno y su representación múltiple.

El objeto de conocimiento se traslada a los géneros de conocimiento vistos como "transiciones en curso, como 'esfuerzos' para orientar las tensiones de cada género de conocimiento hacia una mayor actividad $»^{12}$. De esa productividad inmanente surge la idea de belleza que analizo desde la filosofía spinozista como acción de las leyes de cruce propias de la potencia divina sobre el cuerpo y genuina para las infinitas causas posibles en tanto Deus sive X: causa única de leyes no sometidas

6 «Toda la Ética está preocupada por esa conversión de la noción [forma]: se trata de ir desde el juego no reglado de las formas (parte I) a la naturaleza concebida como elemento de transformación legar (prefacio parte III), pasando por una definición original (parte II) de la cual se desprenden asimismo los principios de una medicina (partes IV y v)». François Zourabichvili, Spinoza. Una fisica del pensamiento, Editorial Cactus, Buenos Aires, 2014, p. 9 y ss.

7 La idea de Naturaleza solidaria con una doctrina de transformación rompe por parte de Spinoza con el modelo mecanicista « $1 .{ }^{\circ}$ potencia de la materia (materia capax), $2 .{ }^{\circ}$ individuación total o partitiva (formas), $3 .^{\circ}$ actualización exhaustiva, luego necesaria, de esa potencia (formas omnes successive assumit)". François Zourabichvili, ibidem.

${ }^{8}$ Balibar, p. 268 y ss.

9 Ibidem.

${ }^{10}$ Digo absolutamente infinito, no solo en su género; pues de aquello que es meramente infinito en su género podemos negar infinitos atributos, mientras que a la esencia de lo que es absolutamente infinito pertenece todo cuanto expresa su esencia, y no implica negación alguna. EI, def. vI, Explicación.

11 «Una vez que los hombres se han persuadido de que todo lo que ocurre, ocurre por causa de ellos, han debido juzgar como lo principal en toda cosa aquello que les resultaba más útil, y estimar como las más excelentes de todas aquellas cosas que les afectaban del mejor modo. De donde han debido formar nociones, con las que intentan explicar la naturaleza de las cosas, tales como Bien, Mal, Orden, Confusión, Calor, Frío, Belleza y Fealdad; y, dado que se consideran a sí mismos como libres, de ahí han salido nociones tales como Alabanza, Vituperio, Pecado y Mérito». EI, Apéndice.

12 Etienne Balibar, op cit., p. 292. 
a exterioridad puesto que son transformación o Dios o Naturaleza. Todo vuelto a la belleza reza el canto de los navajos, materia capax:

Camino con la belleza delante de mí / Camino con la belleza a mis espaldas / Camino con la belleza debajo de mis pies / Camino con la belleza a mi alrededor / Todo a vuelto a la belleza ${ }^{13}$.

Imposibles posibles parecen recrearse en un acto de belleza, bajo la aguda reivindicación que prefiere la reducción a lo imposible o su intemperie a la ignorancia cuya suficiencia es el adorno.

Es más activa la belleza cuanto más ejerce para defender un imposible incotestable. La potencia de la belleza es reactiva así con toda la fuerza de su fragilidad ante la monolítica ignorancia ${ }^{14}$. El fracaso de la ignorancia nunca es percibido por esta y de ahí surge uno de sus grandes peligros y de ahí también la conciencia de fragilidad ante ella. Ornamento impotente a la potencia de lo frágil. Este es un trabajo dependiente de la cuestión de la belleza como acto que paradójicamente no atribuye fealdad sino aviso de la impotencia. "¿Qué contribuye más al ornato y perfección del mundo, que haya espectros o que haya múltiples monstruos, como centauros, hidras, arpías, sátiros, grifos, gnomos y otras muchas necedades por el estilo? Sin duda, el mundo hubiera sido bien adornado, si Dios lo hubiera ordenado y aderezado a gusto de nuestra fantasía y con esas cosas que cualquiera imagina y sueña fácilmente, pero que nadie logra entender jamás ${ }^{15}$.

Si no tenemos medio para probar las leyes, las transformaciones, las causas en definitiva, no es prudencia «la reducción, no a lo imposible, sino a la ignorancia ${ }^{16}$, es importante destacar la fuerza de lo imposible contenida en esa frase. Lo imposible puede ser resuelto. El entendimiento regulador de aquella voluntad contempla la diferencia entre acto de belleza y belleza adjetivada, sigue todas las leyes de cruce enmarcadas en el juego de razonamiento o lógica que busca desvelar no forzando a la Naturaleza. Es una falta a la totalidad lo contrario; de hecho, para el modo humano es asunto crucial verlo en favor del conatus, pues para saber el cuerpo por el cuerpo le urge entender el cruce de la ley en él a la manera que cantan los navajos. Se trata de entender lo humano de la belleza como causa perseverante para la salud tan deseada como escurridiza por su gran dependencia fisicoquímica.

13 Enzzo Tezzi, La belleza y la ciencia. Hacia una visión integradora de la naturaleza, p. 13.

14 «Por lo que toca a las otras nociones, tampoco son otra cosa que modos de imaginar, por los que la imaginación es afectada de diversas maneras, y, sin embargo, son consideradas por los ignorantes como si fuesen los principales atributos de las cosas; porque, como ya hemos dicho, creen que todas las cosas han sido hechas con vistas a ellos, y a la naturaleza de una cosa la llaman buena o mala, sana o pútrida y corrompida, según son afectados por ella. Por ejemplo, si el movimiento que los nervios reciben de los objetos captados por los ojos conviene a la salud, los objetos por los que es causado son llamados bellos; y feos, los que provocan un movimiento contrario». EI, Apéndice

15 Baruj Spinoza. Correspondencia, carta 54. (Edición por Atilano Domínguez, en Guillermo Escolar, Ed. Madrid, 2020).

${ }^{16}$ EI, Apéndice. 


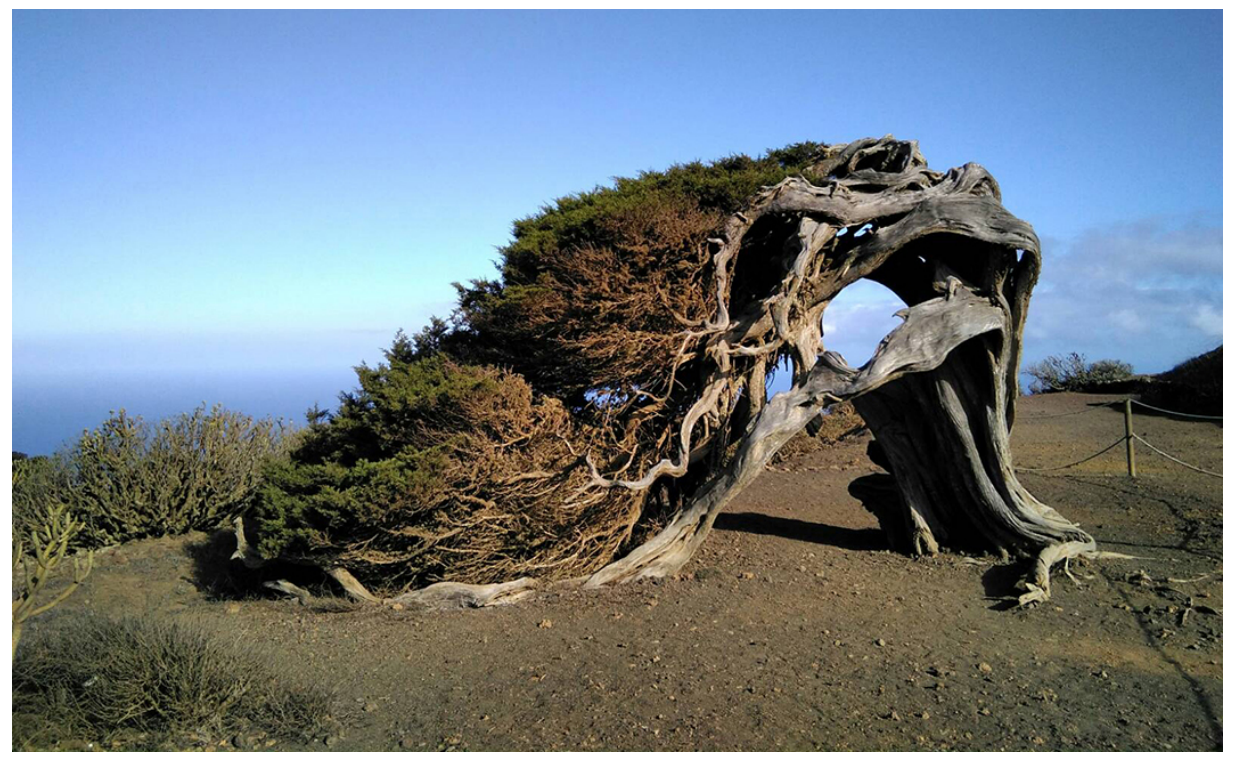

Árbol La Sabina. Isla de El Hierro, Islas Canarias.

Spinoza nos acerca su posición al contestar por qué «cada parte de la naturaleza concuerda con su todo y de qué forma se conecta con lo demás» ${ }^{17}$. Va en su respuesta a Oldenburg el movimiento característico de quien escarba en la pregunta hasta encontrar su núcleo y se sitúa con conciencia ante lo desconocido. Declara lo que ignora sin refugiarse en la ignorancia; eso no admite matiz, como leímos antes. Su ignorancia es parte de su conocimiento visto a la manera socrática:

supongo que usted me pide las razones por las que estamos convencidos de que eso es así. Pues conocer cómo realmente están conectadas y cómo cada una concuerda con su todo, ya he dicho en mi carta anterior que lo ignoraba; puesto que para conocer eso, sería necesario conocer toda la naturaleza y todas sus partes. Procraré, pues, mostrar el motivo que me fuerza a afirmar eso. Pero, previamente quisiera advertir que no atribuyo a la naturaleza belleza o deformidad, orden ni confusión. Porque las cosas no se pueden llamar hermosas o deformes, ordenadas o confusas, sino respecto a nuestra imaginación ${ }^{18}$.

En las sabinas de la isla de El Hierro encontramos formas que cabe interpretar hermosas, deformes, ordenadas, confusas y todo a la vez respecto a nuestra imaginación. Respecto a verla a la luz del día o en la oscuridad de la noche. Las

17 Baruj Spinoza. Correspondencia, carta 32.

18 Ibidem. 
Sabinas se han modificado por la acción del viento en figuras que la mirada de Labordeta describe así:

En la otra parte de la costa, donde los alisios arrebatan la vida, las Sabinas, las enormes y heroicas Sabinas, se enfrentan desde años al arrebatador viento que las tortura y martiriza. Ellas, como damas lorquianas, como trágicas vestales se doblan contra el suelo dejando sus melenas a merced del viento. Son como el dolor árido, como el grito descompuesto, como el gesto de un paisaje emocionante estremecido contra la ronca voz del horizonte ${ }^{19}$.

La belleza torna a ellas en las palabras del cantautor, no es un error la contorsión de las ramas de la Sabina y si lo fuera es un dato a favor de la voluntad informada. La claridad del error también torna a la belleza que actúa por el lenguaje. La Naturaleza se ofrece a la mirada que da con la capacidad de ser afectada y afectar, como lo son las ramas por el viento y nada las hace perfectas sin él. Dicho desde la perspectiva spinozista: «La perfección y la imperfección son denominaciones que no difieren mucho de las denominaciones de belleza y deformidad ${ }^{20}$. Deformidad de un Dios que se amplifica en ella, un rasgo más de la causa sui. Atilano Domínguez comenta la carta 55 escrita por Boxel a Spinoza y puntualiza que ante la «crítica spinoziana de la belleza, como experiencia estética o sensible, Boxel le propone un concepto intelectualista, basado en la armonía o 'proporción' general (supremos géneros) del universo. Pero, en realidad, esta es también una idea de Spinoza, aunque no suele calificarla de 'belleza', aunque sí de 'orden' natural»". El «orden» esencia del arte de la transformación. Expresión de la infinita potencia dentro de la teoría de la imaginación humana de la que nos habla Spinoza.

Si a la luz del Sol o a la luz de la Luna las impresiones son distintas del mismo objeto, tanto más distintas vistas de forma mágica o desde la perspectiva racional. Para Boxel ¿sería la Sabina un fantasma nocturno? (carta 53) los espíritus existen para él entre otros argumentos por las siguientes razones que resumo:

1. Coherente con la belleza y perfección del universo.

2. Verosímil que el creador los haya creado, son más semejantes a él que creaturas corpóreas.

3. Hay cuerpo sin espíritu y por tanto espíritu sin cuerpo.

4. No hay aire o cuerpo oscuro sin habitantes. Luego el espacio inconmensurable está lleno de espíritus que lo habitan. Unos superiores verdaderos espíritus otros inferiores más sutiles e invisibles. Ninguno de sexo femenino.

El primer argumento ya introduce el aspecto refractario de la superstición a la parte que actúa por belleza verbo (su secuencia causal) y no adjetivo. Esto

19 J.L. Labordeta, Con la mochila a cuestas, Editorial RBA, Barcelona, 2001 p. 105.

20 Baruj Spinoza. Correspondencia, carta 54.

${ }^{21}$ Ibidem, carta 55, nota al pie. 
puede ser entendido, sin hablar de estética en la época de Spinoza, a través de la pregunta sobre el arte que desde la semiótica es pertinente hacer. Así pues, «el anacronismo del spinozismo podría revelarse de una actualidad inesperada y de una utilidad inédita $»^{22}$. La secuencia de argumentos resumidos antes da ejemplo de lo que Spinoza critica, es decir, ante ellos su crítica a la ontologización de lo bello confluye con la crítica al finalísmo por una tendencia «demasiado humana estetizar la naturaleza como obra de un supremo arquitecto $»^{23}$, una tendencia que quiebra un acuerdo racional sobre la belleza efectiva con quienes piensan en el universo un ser de la belleza y la perfección. Aquí se señala el abismo entre Spinoza y su interlocutor un abismo de lenguaje.

Su primer argumento es que «es coherente con la belleza y perfección del universo» que haya espíritus. La belleza, distinguido señor, no es tanto la cualidad del objeto que se ve, cuanto un efecto en el sujeto que lo ve. Si nuestros ojos fueran más largos o más cortos, o si nuestro temperamento fuera diferente, las cosas que ahora nos parecen bellas, nos parecerían deformes, y las que ahora nos parecen deformes, nos parecerían bellas. Una mano hermosísima, vista en el microscopio, nos parecerá horrible. Algunas cosas, vistas de lejos son bellas, y vistas de cerca, deformes. De suerte que las cosas, consideradas en sí misma o en relación a Dios, no son ni bellas ni deformes. Por tanto, laque que dice que Dios creó al mundo para que fuese bello, debe admitir una de esas dos alternativas: o bien que Dios formó el mundo para el deseo y los ojos del hombre o bien que formó el apetito y los ojos del hombre para el mundo ${ }^{24}$.

Entender la belleza como perseverancia nos sitúa en una nueva reflexión sobre la naturaleza del arte ligando a él la noción de libertad ${ }^{25}$ entendida como arte de la naturaleza, tomo las palabras de Lorenzo Vinciguerra como prueba para esta defensa del acto de belleza -de la potencia que incluye su significado en movimiento o como motor- en el proceso creativo entendido como arte sin estética ${ }^{26}$ corpóreo $^{27}$ por la salud mencionada y por la sabiduría que lo medita para la libertad. Desde la perspectiva aesthetica sive ethica el autor de La semiótica de Spinoza explica arte concerniente a la ética, que no tiene que ver con la belleza sino con la libertad. Una manera de evitar subordinaciones.

En contra de lo bello como propiedad de las cosas está la representación mental de la belleza. Pensar la belleza en términos de movimiento frente a lo bello

22 Lorenzo Vinciguerra, La semiótica de Spinoza, Cactus, Buenos Aires, p. 175.

${ }^{23}$ Ibidem, p. 176.

${ }_{24}$ Baruj Spinoza. Correspondencia, carta 54.

${ }^{25}$ L. Vinciguerra, op. cit., p. 180.

26 Ibidem, p. 173.

${ }_{27}$ Pertenece a la esencia del arte aquello que el cuerpo puede en virtud de su propia potencia, así como a la filosofía pertenece la naturaleza y el origen del pensamiento. Por esa misma razón no existe verdadera filosofía sin un arte del cuerpo que acompañe su práctica. Juntas constituyen el arte de vivir para el que Spinoza tenía una sola palabra: ética. Ibidem, p. 182. 
como una etiqueta idealista produce efectos que en cada caso abren pensamientos divergentes. La efectiva respuesta de Spinoza no solo se aleja completamente de la estela neoplatónica sino que además se apoya y fundamenta en la naturaleza fisiológica de los receptores que discriminan las entradas saludables y en ocasiones lo contrario. En la discriminación no es aceptable un salto de fe que decida por superstición o amparado en la trascendencia sin un saber latente y práctico ${ }^{28}$. Se fía a la cooperación humana el logro de ese saber sin refugio trascendental ${ }^{29}$.

A manera de resumen. Pensar la naturaleza en toda lo que satisface la idea Natura sive $X$ incluye pensar desde ese mismo lugar en sus conexiones ${ }^{30}$. Este pensamiento integrador acerca de $X$ da sentido inmanente a la creación artística en todas sus relaciones, incluida la conexión a lo imposible como relato sobre la transformación de la forma desde donde entendemos en parte aquello que no se niega a la singularidad de la mente y por lo tanto para su traducción cuerpo. Es esta una dimensión de cautela y cuidado.

La relación de dichas conexiones por las que el Dios de Spinoza se diversifica en sí mismo concede un pase galénico a la belleza enfocada hacia el contento de sí. Un placer que expresa la belleza como acción en el seno de relaciones infinitas. En ellas que se encuentra el significado que otorga pensar en esa acción como una expresión. “Quién diría al ruiseñor que su canto es frívolo por carecer de palabras?»"31. Tal es

28 «Desde la perspectiva epistémica de la estructura del sistema este axioma proporciona la clave de la aplicación práctica o moral al eje conatus-virtus. Un principio regulador que fundamentado en la física permite su aplicación técnica. Es una barrera natural imposible de franquear, cuyo conocimiento autoriza, empero el manejo de las fuerzas de la naturaleza. De su aplicación Spinoza deducirá importantes consecuencias éticas que conciernen: en primer lugar, a las ideas y al conocimiento (E4 p. 1); en segundo lugar, a la acción (lo que puede y lo que no puede el individuo humano; E4 pp. 2 y 4); en tercer lugar, a las emociones y los afectos (E4 pp. 5 y 7; p. 8 y ss.); y finalmente, a los fundamentos de la vida social y política (E4 p. 37 e. II». María Luisa de la Cámara, Métoikos: Spinoza Trágico, Editorial Dickinson S.L., Madrid, 2020, p. 122.

${ }_{29}$ «De tal suerte, no cesarán de preguntar las causas de las causas, hasta que os refugiéis en la voluntad de Dios, ese asilo de la ignorancia. Así también, cuando contemplan la fábrica del cuerpo humano, quedan estupefactos, y concluyen, puesto que ignoran las causas de algo tan bien hecho, que es obra no mecánica, sino divina o sobrenatural, y constituida de modo tal que ninguna parte perjudica a otra. Y de aquí proviene que quien investiga las verdaderas causas de los milagros, y procura, tocante a las cosas naturales, entenderlas como sabio, y no admirarlas como necio, sea considerado hereje e impío, y proclamado tal por aquellos a quien el vulgo adora como intérpretes de la naturaleza y de los dioses. Porque ellos saben que, suprimida la ignorancia, se suprime la estúpida admiración, esto es, se les quita el único medio que tienen de argumentar y de preservar su autoridad». EI, Apéndice.

30 «Por conexión de las partes no entiendo otra cosa, pues, sino que las leyes o naturaleza de una parte de tal manera se ajustan realmente a las leyes o naturaleza de otra parte, que no existe la mínima contrariedad en ellas. En cuanto al todo y las partes, considero a las cosas como partes de algún todo en tanto en cuanto se ajustan realmente unas a otras, de suerte que concuerdan entre sí en la medida de lo posible; por el contrario, en cuanto discrepan entre sí, cada una de ellas forma en nuestra mente una idea distinta de las demás, y se considera por tanto como un todo y no como una parte». E p. 32, Atilano Domínguez.

${ }^{31}$ Mary Oliver, La escritura indómita, Errata Naturae, Madrid, 2021, p. 91 y ss. 
la dimensión de la belleza que se inserta en la lógica como juego de razón en el seno de la Naturaleza o infinitas (X) posibilidades e imposibilidades pensadas. La imposibilidad y posibilidad se suma a todo aquello que cabe ser pensado por la totalidad. Fundamento de la resignificación del cuerpo capaz de pensarse.

El punto de conflicto entre la relación de relaciones abiertas a individualidades que a su vez se combinan, dividen y multiplican; a ideas que a su vez se abren a otras ideas en una constelación infinita. Es el punto de la generación y la destrucción. Aquel en el que la belleza no se contrapone a la deformidad más que en un sentido apreciativo, interpretativo con la que da ejemplo la música buena en un estado de melancolía, mala para el triste y sin sentido para quien no puede oírla $^{32}$. La cuestión tratada es solidaria a la idea de comunidad por un sentido de convenientia que podemos llamar «amor», pero también de igual forma por la convención de mostrar en la suma de potencia la belleza actuante, así que en esa representación no habría que decir que «la comunidad de los seres humano que se aman los unos a los otros es Dios, sino más bien que entre todos ellos constituyen Dios, o producen lo divino" ${ }^{33}$.

Dirigida de entrada nuestra atención hacia los prejuicios el texto de Mary Oliver nos da una dimensión sobre lo que queda después de estar segura de que vivimos. Esta meditación sobre la belleza con el soporte de la filosofía spinozista se da por bien definida con las palabras de la escritora:

La vida es el Niágara, o no es nada. Yo no sería soberana ni de una sola brizna de hierba, mientras pueda se su hermana. Acerco mi rostro al lirio, que se alza por encima de la hierba, y lo saludo desde el tallo de mi corazón. Vivimos y de esto estoy segura, en el mismo territorio, en el mismo hogar, y nuestra luz proviene del mismo farol. Todos somos salvajes, audaces, asombrosos. Y ni uno solo de nosotros es bonito ${ }^{34}$.

AmorNatura o comprensión de formar parte de la naturaleza sin que la razón pida nada contra ella. Cualquier acción derivada siendo naturaleza es belleza sin que la belleza nos sea devuelta por ella.

Ese conflicto tan alejado de lo que constituye Naturaleza únicamente porque la belleza no tiene ningún fin para ella, lo es sin embargo efectivamente para las cosas singulares entre las que se encuentra la singularidad humana cuya producción no es un imperio a parte "separado de las producciones de la naturaleza" ${ }^{35}$.

Dichas singularidades son las marcas desde donde tomar la visión de lo infinito bajo la casi imposible condición de hacerlo con amor intelectual a Dios. Esta será

32 «Pues una sola y misma cosa puede ser al mismo tiempo buena y mala, y también indiferente. Por ejemplo, la música es buena para el que es propenso a una suave tristeza o melancolía, y es mala para el que está profundamente alterado por la emoción; en cambio, para un sordo no es buena ni mala». EIV Prólogo.

33 E. Balibar, op. cit., p. 286.

${ }^{34}$ Mery Oliver, op. cit.

35 L. Vinciguerra, op. cit., p. 175. 
de forma transitoria la belleza como acto la inminencia de lo imposible que permite percibir con abstracción las leyes de la naturaleza ${ }^{36}$, lo bello para mí es esa percepción como forma de sabiduría.

Recibido: junio de 2021; ACEPTADo: julio de 2021

36 Con lo dicho, he explicado la naturaleza de Dios y sus propiedades, a saber: que existe necesariamente; que es único; que es y obra en virtud de la sola necesidad de su naturaleza; que es causa libre de todas las cosas, y de qué modo lo es; que todas las cosas son en Dios y dependen de Él, de suerte qué sin Él no pueden ser ni concebirse; y, por último, que todas han sido predeterminadas por Dios, no, ciertamente, en virtud de la libertad de su voluntad o por su capricho absoluto, sino en virtud de la naturaleza de Dios, o sea, su infinita potencia, tomada absolutamente. 